\title{
Nanoparticle-Based Drug Delivery for Therapy of Lung Cancer: Progress and Challenges
}

\author{
Anish Babu, ${ }^{1,2}$ Amanda K. Templeton,,2 Anupama Munshi, ${ }^{2,3}$ and Rajagopal Ramesh ${ }^{1,2,4}$ \\ ${ }^{1}$ Department of Pathology, University of Oklahoma Health Sciences Center, Oklahoma City, OK 73104, USA \\ ${ }^{2}$ Peggy and Charles Stephenson Cancer Center, University of Oklahoma Health Sciences Center, Oklahoma City, OK 73104, USA \\ ${ }^{3}$ Department of Radiation Oncology, University of Oklahoma Health Sciences Center, Oklahoma City, OK 73104, USA \\ ${ }^{4}$ The Graduate Program in Biomedical Sciences, University of Oklahoma Health Sciences Center, Oklahoma City, OK 73104, USA
}

Correspondence should be addressed to Rajagopal Ramesh; rajagopal-ramesh@ouhsc.edu

Received 26 August 2013; Accepted 28 October 2013

Academic Editor: Haifeng Chen

Copyright (C) 2013 Anish Babu et al. This is an open access article distributed under the Creative Commons Attribution License, which permits unrestricted use, distribution, and reproduction in any medium, provided the original work is properly cited.

\begin{abstract}
The last decade has witnessed enormous advances in the development and application of nanotechnology in cancer detection, diagnosis, and therapy culminating in the development of the nascent field of "cancer nanomedicine." A nanoparticle as per the National Institutes of Health (NIH) guidelines is any material that is used in the formulation of a drug resulting in a final product smaller than 1 micron in size. Nanoparticle-based therapeutic systems have gained immense popularity due to their ability to overcome biological barriers, effectively deliver hydrophobic therapies, and preferentially target disease sites. Currently, many formulations of nanocarriers are utilized including lipid-based, polymeric and branched polymeric, metal-based, magnetic, and mesoporous silica. Innovative strategies have been employed to exploit the multicomponent, three-dimensional constructs imparting multifunctional capabilities. Engineering such designs allows simultaneous drug delivery of chemotherapeutics and anticancer gene therapies to site-specific targets. In lung cancer, nanoparticle-based therapeutics is paving the way in the diagnosis, imaging, screening, and treatment of primary and metastatic tumors. However, translating such advances from the bench to the bedside has been severely hampered by challenges encountered in the areas of pharmacology, toxicology, immunology, large-scale manufacturing, and regulatory issues. This review summarizes current progress and challenges in nanoparticle-based drug delivery systems, citing recent examples targeted at lung cancer treatment.
\end{abstract}

\section{Introduction}

Worldwide lung cancer is the leading cause of cancer-related deaths with a dismal 5 -year survival rate of only $15 \%$ [1]. Every year in the United States approximately 220,000 individuals are diagnosed with lung cancer of which $85 \%$ of the cases are classified as non-small-cell lung carcinoma (NSCLC) [1] while the remaining cases are diagnosed as small-cell lung carcinoma (SCLC). Current treatment strategies are strongly dependent on the type of malignancy and stage at the time of diagnosis but often involve a combination of surgery, chemotherapy, and/or radiation therapy.

Chemotherapy, a first-line treatment option for advanced-stage lung cancer, is often administered intravenously where it circulates throughout the body ultimately locating and destroying cancerous and normal tissues. Standard first-line chemotherapy regimens for lung cancer include platinum-based drugs such as cisplatin and carboplatin. However, platinum-based chemotherapy is riddled with dose-limiting side effects including nephro- and cardiotoxicity, anemia, intestinal injury, and peripheral neuropathy as well as less serious symptoms of uneasiness, nausea, and fatigue. To mitigate many of these untoward effects, platinum drugs are used in combination with other anticancer agents. Combination therapy involving two to three drugs increases the therapeutic effectiveness and reduces the dosage of each individual drug required to produce an observable therapeutic response. Common chemotherapeutic agents for combination therapy include a platinum drug with paclitaxel, gemcitabine, etoposide, or vinblastine. However, like monotherapy, combination therapy is limited by dose-dependent side effects and patient's intolerability 
to the drug combination resulting in cessation of treatment [2]. Additionally, the hydrophobic nature of the majority of the cancer chemotherapeutics makes them poorly water soluble and therefore limits their administration at high doses $[3,4]$. Thus, methods to improve tumor-targeted delivery of chemotherapeutics that will result in increased drug efficacy with improved pharmacological properties and minimal toxicity to normal tissues remain a priority in cancer therapy.

Experimental therapies such as photodynamic therapy (PDT), immunotherapy, and gene therapy provide promising tools to fight lung cancer. In PDT, a photosensitizer activated by laser light reacts with molecular oxygen to form reactive oxygen species that function to annihilate cancer cells [5]. PDT is often used in combination with chemotherapy or surgery. Porfimer sodium, a first-generation photosensitizer, has been used in the treatment of early as well as advanced lung carcinomas. More improved and efficient PDT agents are currently available as a result of the extensive research efforts in the last two decades. However, many of these photosensitizers are poorly water soluble fettering their intravenous administration [6].

Immunotherapy harnesses the body's immune system to fight cancer. Biomolecules or antigens are administered to either trigger the immune system or reduce the immune suppressing activities of the tumor [7]. Administration of immunologically active agents disrupts the tumorigenic cascades by directly blocking growth factors or hormones and their receptors. Certain cancers including lung cancer overexpress growth factor receptors such as the epidermal growth factor receptor (EGFR/Herl). Binding of the ligand epidermal growth factor (EGF) to EGFR activates cell proliferation and survival signaling pathways resulting in rapid and uncontrolled tumor growth. Cetuximab, a competitive anti-EGFR monoclonal antibody, counteracts the cell proliferation signaling mediated by the endogenous EGF ligand culminating in attenuation of the cell survival signals and induction of tumor cell death. Gene therapy is a relatively new concept with a large number of research teams worldwide in active pursuit of identifying and delivering cancer-suppressing genes for clinical applications [8]. Delivery vectors are a necessity in order to protect the therapeutic genes until they reach their target site. Historically, viral vectors have been used to deliver gene-based therapeutics [9]; however, viral vector induced host immune responses limits their therapeutic potential [10]. Definitively, there is a growing need for development of safe and efficient delivery vehicles for photosensitizers, chemotherapeutics, and tumor suppressor genes.

Nanotechnology is not pervaded by some of the limitations of viral vectors providing an avenue of incredible potential for development of tumor-targeting drug delivery systems. This continuously expanding niche will revolutionize cancer treatment and management [11]. More precisely, nanoscale drug delivery systems hold great promise in successfully formulating and enhancing the therapeutic efficacy of a large number of anticancer agents [12]. Nanoparticles are known to positively alter biodistribution increasing therapeutic efficiency and reducing nonspecific toxicity of potent anticancer drugs. Their superior biocompatibility, ability to protect nucleic acids from degradation, and ability to deliver therapeutic genes to cancer cells in vivo make nanoparticles the ideal delivery vehicle $[13,14]$. While many nanoparticlebased therapies have been developed, such as Abraxane, an albumin-bound paclitaxel nanoformulation for the treatment of metastatic NSCLC [15], few have been translated into clinical success. It continues to be a challenge to identify ideal drug delivery systems for several classes of novel drugs with different physicochemical characteristics and varying degrees of therapeutic activities in the physiological environment. This review summarizes current progress and challenges in nanoparticle-based drug delivery systems, citing recent examples of applying nanomedicine for lung cancer treatment.

\section{Progress in Nanoparticle Drug/Gene Delivery Systems}

2.1. Lipid-Based Nanocarriers. Liposomes, oil dispersions (micelles), and lipid nanoparticles are the major classes of lipid-based nanocarriers for drug and gene delivery applications. Liposomes are bilayered phospholipid vesicles commonly used to deliver hydrophobic and hydrophilic drugs through either incorporation in the lipid bilayer itself or encapsulation in the inner aqueous core, respectively. Reduction of the number of lipid bilayers reduces the size of the liposomes to nanosize increasing the circulation time and tumor localization properties of encapsulated drugs [16].

Liposomes are becoming increasingly more popular delivery vehicles for anticancer therapeutics due to their strong biocompatibility properties. Over the last decade, the liposomal research field has boomed generating many new liposomal formulations such as cationic liposomes [17], virosomes [18], temperature-sensitive liposomes [19], and archaeosomes [20]. Despite these huge advances at the bench, currently there are only two FDA-approved liposomal formulations: DOXIL, a liposomal doxorubicin injection for ovarian cancer, and Marqibo, a liposomal vincristine sulphate injection for lymphoblastic leukemia.

In lung cancer treatment, liposomes may be a promising delivery system for drugs and genes. The drug of choice for the treatment of NSCLC for the last two decades, cisplatin, is implicated in the development of nephrotoxicity in $20 \%$ of patients receiving high doses [21]. In 2004, Boulikas developed a liposome-based cisplatin drug called Lipoplatin to reduce systemic toxicity of cisplatin [22]. Furthermore, these researchers also demonstrated that lipoplatin injection compared to standard therapy significantly reduced nephrotoxicity to negligible levels in multiple rat tumor models [23]. According to a recent report, lipoplatin is anticipated to complete phase III clinical trial testing in 2013 and 2014 [24]. Paclitaxel, another chemotherapeutic drug widely used in the treatment of lung cancer, was historically formulated using Cremophore EL to enhance its solubility in physiological fluids. However, this resulted in hypersensitivity reactions complicating its systemic delivery. In 2010, a phase I clinical trial in NSCLC patients with malignant pleural effusions demonstrated in all cases investigated that treatment with 
paclitaxel formulated with a liposomal carrier had enhanced therapeutic efficacy [25]. Moreover, a recent preclinical study has shown that liposomal-paclitaxel formulation can be modified to target lung cancer cells to reduce the incidence of drug resistance [26]. Specifically, the liposomal surface was decorated with the mitochondrial targeting molecule d- $\alpha$-tocopheryl polyethylene glycol 1000 succinatetriphenylphosphine conjugate (TPGS1000-TPP). These targeted paclitaxel liposomes could significantly enhance their cellular uptake inducing mitochondria-mediated apoptotic cell death in human A549 lung cancer cells. At present Lipusu, a paclitaxel-liposome, is commercially available with several other formulations under clinical investigation [27]. Table 1 lists current examples of liposomal formulations undergoing clinical trials intended for the treatment of cancer. In a randomized phase III multicenter trial, liposomal formulation of cisplatin and paclitaxel combination therapy reached effective therapeutic response while reducing nephrotoxicity in NSCLC patients [28]. Interestingly, this liposomal drug combination is reported to not only improve the targeting efficiency to the primary tumor but also be effective against metastasis.

Liposomes have also been used to deliver cancer vaccines for the prevention or treatment of existing cancers. Studies using therapeutic vaccine Biomira Liposomal Protein 25 (BLP25) have shown encouraging results in the treatment of advanced NSCLC [29]. BLP25 uses a liposomal carrier that targets the tumor-associated antigen MUC1 to prevent tumor growth. A preclinical study in a human MUC1 transgenic lung cancer mouse model (hMUC1.Tg) demonstrated that pretreatment with a low dose of cyclophosphamide followed by two cycles of liposome BLP25 treatment significantly reduced the number of tumor foci [30]. Importantly, phase III clinical studies using liposome BLP25 are currently underway [31].

Studies from our own laboratory have shown that lipid based nanocarriers can be effectively used for gene delivery in mouse lung cancer models. Preclinical studies using the nontargeted nanoparticle system 1,2-dioleoyl-3Trimethylammonium Propane (DOTAP):cholesterol (Chol.) carrying tumor suppressor genes such as p53, TUSC2/FUS1, or $m d a-7 / I L-24$ [32] efficiently delivered therapeutic genes to metastatic tumor sites culminating in a significant therapeutic effect with increased animal survival. Preclinical studies from our laboratory demonstrating efficacy and safety of the DOTAP:Chol. nanoparticle system resulted in its clinical testing for delivery of the TUSC2/FUS1 tumor suppressor gene in NSCLC patients. Results from the phase I clinical trial demonstrated that intravenous administration of TUSC2 encapsulated in our DOTAP:Chol. nanoparticle system was safe and well tolerated with no treatment-related toxicity. Additionally, study results showed that the nanoparticles were efficiently taken up by primary and metastatic tumors, expression of transgene and gene products occurred, and specific alterations in TUSC2-regulated signaling pathways were observed [32]. Results from this trial have led to discussion for initiating a phase II study for lung cancer. Additional phase I trials testing DOTAP:Chol.-based nanoparticle therapy for breast, ovarian, and pancreatic cancers are anticipated.
The therapeutic genes to be delivered will vary and depend on the cancer type.

Solid lipid nanocarriers (SLNs) are another class of vehicle for drug and gene delivery. SLNs are superior to their lipid counterparts in their enhanced stability, high drug loading, improved biocompatibility, and ease of large-scale manufacturing production. Choi et al. [33] transfected p53-null H1299 lung cancer cells with SLN-carrier $p 53$. The authors were able to demonstrate efficient p53 protein expression compared to commercially available Lipofectin suggesting that SLNs could be used as highly efficient gene therapy vehicles in lung cancer. In a recent report, researchers successfully loaded SLNs with Bcl-2 siRNA and paclitaxel for synergistic combination therapy as well as coencapsulated CdSe/ZnS quantum dots to bestow optical traceability [34]. Collectively, the properties of SLNs are ideally suited for combined chemoand/or gene-therapy and molecular imaging of cancer.

2.2. Polymeric Nanoparticles. As the name suggests, polymeric nanoparticles are synthesized from polymers. More recently, biodegradable polymers such as poly(lactic acid) (PLA), poly(lactic-co-glycolic) acid (PLGA), gelatin, albumin, chitosan, polycaprolactone, and poly-alkyl-cyanoacrylates have gained popularity in use because of their controlled and sustained release properties, subcellular size, and biocompatibility. For instance, Abraxane, an FDA-approved albumin-based nanoparticle carrying paclitaxel, is indicated for first-line treatment of locally advanced or metastatic NSCLC in combination with carboplatin in patients who are not candidates for curative surgery or radiation therapy. Polymer nanoparticles have been shown to enhance the chemo- and radio-therapeutic efficacy of anticancer agents [35]. Chemoradiation therapy involves the concurrent administration of chemotherapy and radiotherapy for the treatment of many cancers, including lung cancer. Chemoradiation therapy is known to improve the local tumor control and patient survival. Polyethylene glycol- (PEG-) modified polylactic acid nanoparticles loaded with taxanes have significantly improved the efficacy of chemoradiation therapy in both in vitro and in an A549 lung tumor xenograft model [36]. Other research groups have developed a cremophorfree nanoformulation of paclitaxel and cisplatin using block copolymers of PEG and polylactic acid for the treatment of lung cancer [37]. This nanoformulation called Genexol-PM has entered phase II clinical trials in patients with advanced NSCLC. A separate phase II clinical trial is awaiting results for the same nanocarrier modified to deliver gemcitabine to untreated patients diagnosed with metastatic lung cancer [38].

Traditional anticancer agents are loathed for their repugnant side effects including the discomfort and pain associated with their administration. Historically, oral drug delivery methods have not been feasible for the treatment of lung cancer due to the inability of the therapeutic to penetrate lung tumor sites and achieve efficient therapeutic concentration even at high administered doses. The size, shape, and surface charge of nanoparticles provide an avenue for the development of novel routes of administration for anticancer agents. Recently, Jiang et al. [39] investigated three different 
TABLE 1: Ongoing or recently completed clinical trials of a few liposomal nanoformulations used for cancer chemotherapy* .

\begin{tabular}{|c|c|c|c|}
\hline Trade name/composition & Indication & Phase & Stage \\
\hline PEGylated Liposomal Doxorubicin & AIDS-associated non-Hodgkin's lymphoma & I & Completed \\
\hline Doxil/CAELYX Liposomal Doxorubicin & $\begin{array}{l}\text { Malignant female reproductive system neoplasm, Ovarian } \\
\text { cancer }\end{array}$ & I & Active \\
\hline Doxil Liposomal Doxorubicin & Resistant solid malignancies & I & Completed \\
\hline Liposomal Cytarabine & $\begin{array}{l}\text { Central nervous system malignancies, } \\
\text { Stage IV breast cancer }\end{array}$ & II & Active \\
\hline $\begin{array}{l}\text { Liposomal Entrapped Paclitaxel Easy to } \\
\text { Use (LEP-ETU) }\end{array}$ & Advanced cancer & I & Completed \\
\hline Liposomal Daunorubicin & $\begin{array}{l}\text { Hematologic cancer, } \\
\text { Chronic myelomonocytic leukemia } \\
\text { previously treated Myelodysplastic syndromes and } \\
\text { recurrent adult Acute myeloid leukemia }\end{array}$ & II & Completed \\
\hline BLP25 Liposome Vaccine & $\begin{array}{l}\text { Lung neoplasms } \\
\text { Non-small-cell lung carcinoma }\end{array}$ & II & Completed \\
\hline $\begin{array}{l}\text { CPX-351 } \\
\text { Liposomal Cytarabine-Daunorubicin }\end{array}$ & Acute myeloid leukemia & I & Active \\
\hline Liposomal Vincristine & Acute lymphoblastic leukemia & II & Active \\
\hline Liposomal LE-SN38 & Advanced cancer & I & Completed \\
\hline $\begin{array}{l}\text { IHL-305 } \\
\text { Irinotecan Liposome Injection }\end{array}$ & Advanced solid tumours & I & Active \\
\hline $\begin{array}{l}\text { Liposome Encapsulated Mitoxantrone } \\
\text { (LEM) }\end{array}$ & Advanced cancer & $\mathrm{I}$ & Completed \\
\hline $\begin{array}{l}\text { Liposomal Encapsulated Docetaxel } \\
\text { (LE-DT) }\end{array}$ & Advanced solid tumours & I & Completed \\
\hline
\end{tabular}

${ }^{*}$ Data retrieved from US National Institutes of Health website (http://clinicaltrials.gov/) on August 21, 2013.

polymer-based nanoparticles composed of polycaprolactone (PCL) that were surface modified with chitosan polymer for oral administration of chemotherapy in lung cancer. The mucoadhesive properties of the polymer increased the therapeutic effect of anticancer drugs by selectively interacting with the increased levels of mucin expressed in cancer cells compared to normal cells. Similarly, another study reported the use of PCL-based diblock copolymer nanoparticles for treating lung cancer via oral administration [40]. Interestingly, this nanoparticle showed advantages over the commercially available Taxotere, an injectable docetaxel, in terms of cytotoxicity against lung cancer cells. Another research group designed a chitosan-based controlled drug delivery system to deliver the potent antineoplastic agent lomustine. The lomustine-loaded chitosan nanoparticles demonstrated excellent control over drug release and enhanced its in vitro cytotoxicity against the lung cancer cell line L132 [41]. Recently, attention has turned towards polymer nanoparticles with expansile properties for the treatment of lung cancer. A Lewis lung carcinoma mouse model demonstrated that following surgical intervention paclitaxel-loaded expansile nanoparticles delayed the local recurrence of subcutaneous lesions as well as modestly improved the overall survivability of the tumor-bearing animals [42].

The advantage of combining PLA or PLGA nanoparticles with chitosan has recently materialized. Chitosan modification of drug- or gene-loaded PLGA nanoparticles imparts a positive charge to the nanoparticle surface, which aids in cellular uptake and cytotoxicity towards lung cancer cells $[43,44]$. Identifying the potential of chitosan-modified nanoparticles as drug or gene delivery vehicles provides an opportunity for combined chemo- and gene-therapeutic approaches with same or similar kinds of nanoparticle systems. Currently, our lab is developing a chitosan/PLAhybrid-based nanoparticle system for combined delivery of chemotherapeutics and tumor suppressor genes for lung cancer. The nanoparticles are designed such that the rigid PLA polymer matrix containing the chemotherapeutic drug forms the inner core and is surface coated with siRNA or DNA containing chitosan and decorated with tumortargeting moieties. The nanoparticle is less than $200 \mathrm{~nm}$ in size and is physicochemically stable for at least 10 days in solution as well as having a drug encapsulation efficiency greater than $90 \%$ (data unpublished). The ultrastructure of this drug-loaded nanoparticle system is shown in Figure 1.

Polymer nanoparticles have been extensively used in studies aimed at delivering targeted chemotherapeutics to lung cancer. Tseng et al. [45] developed a gelatin nanoparticle system decorated with EGFR-targeted biotinylated EGF (bEGF). These nanoparticles demonstrated enhanced cellular uptake in EGFR overexpressing cancer cell lines holding promise for targeted lung cancer therapy. Building on this strategy, the same group reported an aerosol-targeted therapy in a mouse model for lung cancer using bEGF-gelatin nanoparticles loaded with cisplatin [46]. The aerosol-based targeted drug delivery system resulted in enhanced drug 


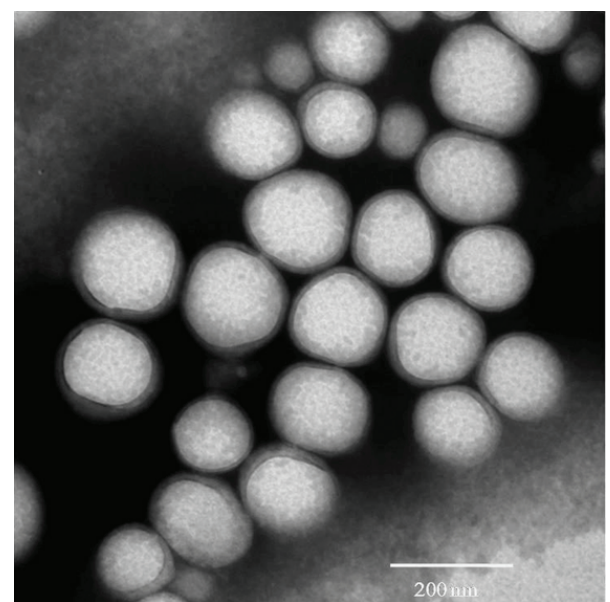

Figure 1: Transmission electron microscopy of cationic polymer coated poly (lactic acid) nanoparticle carrying the anticancer drug cisplatin.

concentrations in tumor tissues contributing to anticancer activity while direct tumor injection with the nanoparticles yielded high therapeutic efficacy and reduced systemic toxicity of cisplatin. Additionally, gelatin nanoparticles have also been used for the delivery of the hydrophobic drug resveratrol towards NSCLC cells [47].

Dendrimers are branched polymers with a large number of functional groups that radiate from a central core providing the opportunity to link multiple bioactive molecules. A recent study illustrated the use of dendrimer-targeting peptide conjugates as a carrier for drugs towards NSCLC [48]. These dendrimer-peptide conjugates when administered to a lung tumor-bearing athymic mouse model were efficiently taken up by the cancer cells demonstrating their potential as a drug carrier for the treatment of lung cancer [48]. In a related study, a newly designed PEGylated dendrimer nanoparticle showed promising application as an aerosol-inhaled drug delivery modality [49]. The smaller dendrimer particles are reported to enter the blood stream via inhalation while larger particles are sequestered in the lung for an extended period of time. In the future, this method of controlled drug delivery to the lungs could provide an alternative to injectable drug systems. Starpharma Holdings Ltd., Australia, released the primary data of a study utilizing delivery of dendrimer-based doxorubicin to rats burdened with metastatic breast cancer in the lungs [50]. The authors concluded that there was substantial improvement in the efficacy of doxorubicin when delivered using dendrimers.

Hybrid polymer nanoparticles composed of PLGA and chitosan demonstrated enhanced tumor uptake and cytotoxicity compared to unmodified nanoparticles in A549 cells [51]. More importantly, the modified nanoparticles administered to a lung metastatic mouse model demonstrated a lungspecific increase in biodistribution [51]. PLGA nanoparticles have also successfully been used to codeliver paclitaxel and STAT3 siRNA to the drug-resistant A549 cell line [52]. In another study, paclitaxel-loaded PLGA nanoparticles decorated with anti-EGFR demonstrated high binding affinity to EGFR expressing cells in a mouse lung tumor model indicating the potential of these nanoparticles for targeted lung cancer therapy [53].

2.3. Metal-Based Nanoparticles. Noble metals such as gold and silver have been extensively investigated for clinical applications, including their use in sensitive diagnostic imaging, detecting, and classifying of lung cancer [54]. Peng et al. [55] developed a gold nanoparticle-based biosensor system with the capacity to detect lung cancer by analyzing an individual's exhaled breath. The sensor uses a combination of an array of chemiresistors based on gold nanoparticles and pattern recognition methods. Additionally, another research group reported the detection of picograms of enolase 1 (ENO1), an immunogenic antigen associated with NSCLC, by using an immunosensor that detects electrochemical signal probes of gold nanoparticle congregates [56]. Recently, gold nanoparticles have also successfully been tested as sensors for discriminating and classifying different lung cancer histologies. The sensor was able to distinguish between normal and cancerous cells, SCLC and NSCLC, and between two subtypes of NSCLCs [57].

Additionally, gold nanoparticles have been used to deliver anticancer drugs for enhanced therapeutic effectiveness. For example, methotrexate (MTX) has poor tumor retention ability due to its high water solubility, which likely contributes to its slow or poor therapeutic response in patients. However, gold nanoparticle conjugates of MTX have high tumor retention and enhanced therapeutic efficacy in a Lewis lung carcinoma mouse model [58]. Previously, we have shown in NSCLC cells that anti-EGFR antibody (Clone 225) conjugated hybrid plasmonic magnetic nanoparticles exhibited significant enhancement in anticancer activity by inducing autophagy and apoptosis [59]. Other studies have developed gold/iron oxide nanoclusters surface decorated with fluorescently labeled antibodies for targeting EGFR expressing epidermoid carcinoma cells [60]. Such design provides promising applications of gold-based nanoparticles in simultaneous use in magnetic resonance imaging (MRI) and therapy. Recently, gold nanoparticles have been applied in PDT for delivery of the water soluble PDT agent purpurin-18$\mathrm{N}$-methyl-D-glucamine (Pu-18-NMGA) towards A549 cells [61]. PDT using Pu-18-NMGA-gold nanoparticle resulted in higher photodynamic activity than free Pu-18-NMGA. Similarly, silver nanoparticles have demonstrated antiproliferative effects in cancer cells $[62,63]$. However, in vitro exposure of human lung cancer cells to silver nanoparticles resulted in reactive oxygen species-induced genotoxicity raising concerns of an unfavorable risk to benefit ratio [64].

2.4. Other Nanoparticle Systems. Magnetic nanoparticles have been extensively investigated and applied in diagnosis and treatment of various cancers. Theranostic nanoparticles concurrently facilitate imaging and delivery of therapeutic agents. Magnetic hyperthermia is a noninvasive therapeutic approach for lung cancer that entails the heat-induced ablation of desired tumor tissue. When subjected to alternating currents the magnetic material, such as superparamagnetic 
iron oxide (SPIO), nanoparticles generate sublethal heat that causes local tissue damage. Sadhukha et al. [65] evaluated in a mouse model the effectiveness of tumor-targeted SPIO nanoparticles for hyperthermic destruction of NSCLC. The EGFR-targeted SPIO nanoparticles showed enhanced tumor retention and significantly inhibited lung tumor growth.

Wang et al. [66] developed magnetic nanoparticles capable of detecting micrometastasis in lung cancer. Magnetic nanoparticles conjugated with the epithelial tumor cell marker pan-cytokeratin efficiently isolated circulating tumor cells (CTCs) from patients diagnosed with lung cancer. The cells were further identified using quantum dots (Qdots) coupled to the NSCLC micrometastasis marker lungspecific X protein (LUNX) and surfactant protein-A(SP-A) antibody. This is the first study that reported the detection of micrometastasis in peripheral blood of lung cancer patients. Magnetic nanoparticles have also been used to overcome drug resistance. A cisplatin-resistant A549 lung tumor xenograft model was chemosensitized with cisplatin loaded magnetic nanoparticles. Molecular studies demonstrated that cisplatin-loaded magnetic nanoparticle-treated tumors had a significant reduction in localization of lung resistance related proteins and enhanced cytotoxicity of cisplatin [67].

Mesoporous silica nanoparticles (MSNs) have been increasingly used in anticancer drug delivery research due to their dynamic capacity for drug loading, controlled drug release property, and multifunctional ability. Human lung cancer cells primarily take up MSNs by endocytosis [68]. MSNs have also been developed as a carrier for radionuclide isotope holmium-165 $\left(\mathrm{Ho}^{165}\right)$ and tested in a xenograft tumor model [69]. In this model, MSNs were able to hold the radionuclide without release and withstand long irradiation times. Additionally, $\mathrm{Ho}^{165}$-carrying MSNs predominantly accumulated in the tumor tissue following intraperitoneal administration in tumor-bearing mice. Importantly, MSNs enhanced the radio-therapeutic efficacy of $\mathrm{Ho}^{165}$ and demonstrated their potential in managing ovarian cancer metastasis. MSNs have also been developed for inhalation treatment of lung cancer [70]. The elegant nanoparticle system design fully exploited the dynamic drug loading capabilities and multifunctionalization of MSNs. It was designed to simultaneously carry cisplatin, doxorubicin, and two different siRNAs targeted to MRP1 and Bcl-2. Moreover, it was surface decorated with luteinizing hormone releasing hormone (LHRH) peptide for targeted lung cancer therapy. The nanoparticle system carrying siRNA inhibited targeted mRNA causing suppression of cellular resistance to the chemotherapeutics accomplishing an enhanced therapeutic efficacy of cisplatin and doxorubicin. MSNs were also explored in targeted EGFR-based therapies. MSNs modified with the cationic polymer polyethyleneimine (PEI) and surface attached with EGFR ligands selectively targeted EGFR overexpressing NSCLC cells [71]. Moreover, these nanoparticles when loaded with the anticancer agent pyrrolidine- 2 had enhanced targeting and therapeutic efficiencies compared to free drug in a subcutaneous lung cancer model.

\section{Challenges for Nanoparticle-Based Drug Delivery in Lung Cancer Therapy}

The past decade has witnessed tremendous growth and development of drug delivery technology utilizing nanoparticle systems. It is expected that the ongoing research efforts in nanomedicine will continue to lead towards safe, efficient, and feasible drug delivery and highly sensitive and improved imaging agents for diagnostic and disease monitoring applications. However, nanomedicine research is facing numerous challenges in bridging rapidly developing novel ideas and translating them into clinical practice. Synthesizing nanoparticle drug delivery systems has always been complicated by designing an appropriate size to carry effective drug/gene payload and ability to target to the right place. Inappropriate size distribution, undefined structure/shape, poor biocompatibility, and improper surface chemistry are possible risk factors in the biological environment. It has been operose to devise the ideal nanoparticle system for drug delivery to the lungs due to the variability in the physicochemical properties and biological behavior of the particles. A number of obstacles including immune reaction, rate of clearance from circulation, efficiency in targeting, and ability to cross biological barriers will follow when these nanoparticle systems enter the preclinical and clinical testing arenas. Having a solid understanding of the biological behavior of nanoparticles is imperative to achieve the highest drug delivery efficiency.

Identification of Physicochemical parameters are absolutely critical in determining the particle-particle interaction within a biological environment, aggregation tendencies, adsorption of proteins on nanoparticle surface, and intracellular trafficking of nanoparticles. A substantial variation in any of these factors can contribute to poor drug delivery, loss of therapeutic efficiency, and/or toxicity. Thus, the efficacytoxicity balance of nanoparticle systems largely depends on their physicochemical properties. Particles larger than $500 \mathrm{~nm}$ are not recommended for intravenous administration since these particles are rapidly eliminated from the circulation. The ideal nanoparticle for delivering conventional therapeutics to solid tumors is less than $200 \mathrm{~nm}$ in size with a spherical shape and a smooth texture in order to easily transport through tumor vasculature and into tumor cells. Such physical characteristics are likely advantageous to the nanoparticles in exploiting the enhanced permeation and retention (EPR) effect associated with solid tumors. Passively targeted nanoparticles enter through leaky vasculature of the solid tumors and are retained in the tumor tissue for extended periods of time due to impaired lymphatic flow. This unique microphysiology of tumors is exploited by many FDA-approved nanoformulations such as Doxil and Abraxane. In solid tumors, such as lung cancer, the EPR effect plays an important role in determining the efficacy of the nanoparticle-based drug delivery system [72]. The presence of a highly fenestrated blood vasculature in the tumor facilitates the EPR effect allowing the enhanced entry of colloidal nanoparticles into the tumor (Figure 2). Additionally, the poor lymphatic flow in the tumor tissue adds to this effect and results in enhanced retention of 


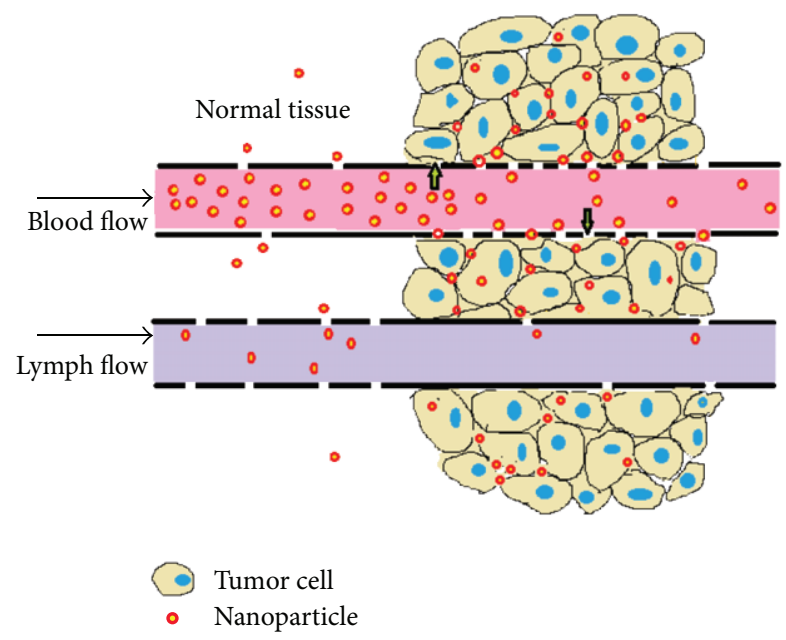

FIGURE 2: A schematic representation of the EPR effect. The leaky vasculature and dysfunctional lymphatics of solid tumors allow the preferential accumulation and retention of colloidal nanoparticles in contrast to the tight vasculature of normal tissue, which excludes nanoparticles.

nanoparticles within the tumor site. In contrast to tumor tissues, the blood vasculature in normal tissues is intact and less permeable attenuating the uptake of nanoparticles by normal tissues. Furthermore, the size, shape, and surface properties of nanoparticles are critically important for passive targeting of solid tumors. The EPR effect usually applies to particles that are less than $200 \mathrm{~nm}$ in size. However, particles less than $50 \mathrm{~nm}$ in size frequently undergo extravasation from the tumor through the fenestrations and are thus less likely to be retained in the tumor tissue for extended periods of time. Moreover, active targeting of nanoparticles is accomplished by decorating the surface of the nanoparticle with specific ligands to promote the binding and interaction with overexpressed protein receptors on cancer cell surfaces. This approach leads to preferential binding, uptake, and intracellular accumulation of the drug or gene in the targeted cells. However, the overall tumor accumulation and therapeutic effect of targeted nanoparticles may be principally controlled by the EPR effect [73].

Fabrication of polymeric nanoparticles with uniform and sub-200 nm size requires critical control over each and every step in the synthesis procedure, which is always challenging. The size distribution of liposomes or vesicular nanoparticles can be narrowed using common extrusion procedures. However, burst release of the drug and poor stability layer additional challenges in the development of such nanoparticles.

Surface charge determines the fate of nanoparticles in vivo. Particle-particle interactions and aggregation tendencies are largely dependent on the zeta potential of the nanoparticles. Positively charged nanoparticles have an increased affinity for the negatively charged cellular membranes of all cells in the body. Most nanoparticles designed for gene delivery applications use positively charged polymers or lipids to achieve enhanced DNA to nanoparticle interaction. Unfortunately, some cationic nanoparticles have enhanced hemolytic properties inciting concern for their safe use in gene delivery. For example, cationic lipid composition of N [1-(2,3-dioleyloxy) propyl]$\mathrm{N}, \mathrm{N}, \mathrm{N}$-trimethylammonium chloride and dioleoylphosphatidylethanolamine (DOTMA/DOPE) are reported to be highly hemolytic while 3 beta- $\left[\mathrm{N}-\left(\mathrm{N}^{\prime}, \mathrm{N}^{\prime}\right.\right.$-dimethylaminoethane)-carbamoyl]-cholesterol (DC-chol)/DOPE liposomes are moderately hemolytic [74].

Biocompatible polymer nanoparticles are an alternative to cationic lipids for the charge-specific delivery of drugs and/or genes. However, little is known in lung tumor models about the effect of surface charge on biodistribution of nanoparticles. PEGylation is routinely used to mask the surface charge effectively camouflaging the nanoparticle from opsonin proteins and significantly extending the half-life of the nanoparticle in the circulation. Another important issue in drug delivery is nanoparticle stability. Poor stability of nanoparticle systems has been attributed to their aggregation tendencies in the physiological environment. Once aggregated, it is virtually impossible to redisperse the particles into their original distribution pattern. While shear forces can be used to redisperse the particles, this may lead to enhanced drug leaching from the particles and ultimately affect the drug loading and therapeutic efficiencies.

Additionally, further consideration must be given to the complexity of nanoparticles and how this may have a negative impact on drug delivery. Multifunctional nanoparticles are hot topics in the field of nanomedicine [75]. A nanoparticle with a large number of surface functional groups provides an avenue for the attachment of multiple kinds of biomolecules for targeted drug delivery and diagnostic applications for lung cancer. A careful analysis of these nanoparticle systems, however, is necessary prior to testing in an in vivo system. Multifunctionalization generally increases the complexity of the nanoparticle. While a large number of multifunctional nanoparticles, such as theranostic systems targeted to lung cancer are under development, Aurimune (CYT-6091) is currently the only known multifunctional nanoparticle system with diagnostic and therapeutic properties that has entered the clinical setting [76]. Aurimune consists of tumor necrosis factor (TNF), a tumor growth inhibiting agent, bound to colloidal gold nanoparticles for simultaneous imaging and therapy.

Interestingly, increased scientific contributions to the field of nanomedicine have resulted in the emergence of a new area of research, nanotoxicology [77]. Even though nanoparticles of various compositions have displayed strong therapeutic properties towards various types of cancer in preclinical studies, they also carry the risk of inducing toxicity to normal cells. Emphases in particular towards inorganicand metal-based nanoparticles have demonstrated that some nanoparticles induce toxicity to normal cells. For example, silica nanoparticles in their amorphous state have the potential to cause inflammatory reactions on target organs resulting in apoptotic cell death [78]. Thus, the use of silicabased nanoparticles for cancer therapy is limited to low concentrations $(0.1 \mathrm{mg} / \mathrm{mL})$ in vitro [78]. The toxicity of titanium oxide $\left(\mathrm{TiO}_{2}\right)$ nanoparticles towards healthy cells is also a 
matter of concern considering its biological applications [79]. Carbon nanotubes (CNTs) have also been reported to exhibit toxicity to normal cells. CNTs upon interaction with live cells generate reactive oxygen species causing mitochondrial dysfunction and lipid peroxidation [80]. Therefore, stringent in vitro and in vivo toxicity studies for each of the novel nanoparticle systems must be conducted to ensure safety prior to their application in humans.

\section{Conclusion and Future Perspectives}

Nanoparticle-based medicine has infinite potential with novel applications continuously being developed for use in cancer diagnosis, detection, imaging, and treatment. These systems are already helping to address key issues with traditional anticancer agents such as nonspecific targeting, low therapeutic efficiencies, untoward side effects, and drug resistance as well as surpassing their predecessors with the ability to detect early metastasis. The ability of nanoparticles to be tailored for a personalized medicine strategy makes them ideal vehicles for the treatment of lung cancer. Numerous nanoparticle-based experimental therapeutics for lung cancer utilize a combinatorial approach balancing the design with targeting and tracking moieties and anticancer agents. In general, nanoparticles with multicomponent structures allow design flexibility in drug delivery of poorly water soluble molecules as well as imparting the ability to overcome biological barriers and selectively target desired sites within the body.

However, many challenges must be overcome in order to expedite the translation of nanoparticle-based therapies from the bench to the bedside. Nanomedicines are threedimensional structures of multiple components with preferred spatial arrangement to impart their function. Subtle changes in the synthesis process or composition of the complex that alter the physical and/or chemical properties can have adverse effects resulting in pharmacological and immunological challenges. Pharmacokinetics (PK) and biodistribution are known to be effected by small compositional differences in nanoparticles [81]. Moreover, nanoparticles oftentimes require higher bioavailability than traditional small molecules since they are routinely designed for novel routes of delivery such as by nasal or oral administration. In vivo clearance of nanoparticles and release kinetics of the active drug are also complicated by their physical and chemical properties. PEGylation is oftentimes used to mask the nanoparticle to evade detection by macrophages and avoid opsonization and destruction. This strategy effectively increases the circulation time enhancing the distribution and accumulation of nanoparticles at the intended target site. Ultimately, small molecules are cleared by the kidney while larger particles are cleared by Kupffer cells and macrophages in the liver and spleen [82]. Researches are also challenged with nanoparticle induced immune responses, which can be elicited by the nanocarrier, the payload, or both [83]. These findings enumerate the importance for identifying key characteristics of each component and developing a thorough understanding of the physicochemical properties to ensure high reproducibility throughout the formulation process and minimize pharmacological and immunological challenges.

Aside from the difficulty of nanoparticle design and discovery, scientists must also battle the lack of standards in the examination of nanomedicines, including manufacturing processes, functional testing, and safety measurements. Conceptually nanoparticle-based therapy must overcome the same hurdles faced by any new drug: optimal design of components and properties, reproducible manufacturing processes, institution of analysis methods for sufficient characterization, favorable pharmacology and toxicity profiles, and demonstration of safety and efficacy in clinical trials. Standard drugs are usually composed of a single active agent. Nanoparticles are complex in nature with multiple active components that can affect the pharmacological behavior. Such complexity necessitates the modification of standard testing of pharmacokinetic, bioequivalence, and safety measurements. There is an immediate need for regulatory agencies to develop an exhaustive list of tests and a streamlined approval process to proactively address the emergence of new products based on new technologies and facilitate nanomedicine delivery to the clinic.

\section{References}

[1] S. S. Ramalingam, T. K. Owonikoko, and F. R. Khuri, "Lung cancer: new biological insights and recent therapeutic advances," CA Cancer Journal for Clinicians, vol. 61, no. 2, pp. 91-112, 2011.

[2] L. C. Pronk, G. Stoter, and J. Verweij, "Docetaxel (Taxotere): single agent activity, development of combination treatment and reducing side-effects," Cancer Treatment Reviews, vol. 21, no. 5, pp. 463-478, 1995.

[3] J. Lu, M. Liong, J. I. Zink, and F. Tamanoi, "Mesoporous silica nanoparticles as a delivery system for hydrophobic anticancer drugs," Small, vol. 3, no. 8, pp. 1341-1346, 2007.

[4] A. Kumar, S. K. Sahoo, K. Padhee et al., "Review on solubility enhancement techniques for hydrophobic drugs," International Journal of Comprehensive Pharmacy, vol. 3, no. 3, pp. 1-7, 2011.

[5] P. Agostinis, K. Berg, K. A. Cengel et al., "Photodynamic therapy of cancer: an update," CA Cancer Journal for Clinicians, vol. 61, no. 4, pp. 250-281, 2011.

[6] A. Babu, J. Periasamy, A. Gunasekaran et al., "Polyethylene glycol-modified gelatin/polylactic acid nanoparticles for enhanced photodynamic efficacy of a hypocrellin derivative in vitro," Journal of Biomedical Nanotechnology, vol. 9, no. 2, pp. 177-192, 2013.

[7] M. Dougan and G. Dranoff, "Immune therapy for cancer," Annual Review of Immunology, vol. 27, pp. 83-117, 2009.

[8] M. A. Kay, "State-of-the-art gene-based therapies: the road ahead," Nature Reviews Genetics, vol. 12, no. 5, pp. 316-328, 2011.

[9] M. Giacca and S. Zacchigna, "Virus-mediated gene delivery for human gene therapy," Journal of Controlled Release, vol. 161, no. 2, pp. 377-388, 2012.

[10] S. Nayak and R. W. Herzog, "Progress and prospects: immune responses to viral vectors," Gene Therapy, vol. 17, no. 3, pp. 295304, 2010.

[11] J. H. Grossman and S. E. McNeil, "Nanotechnology in cancer medicine," Physics Today, vol. 65, pp. 38-42, 2012. 
[12] A. Z. Wang, R. Langer, and O. C. Farokhzad, "Nanoparticle delivery of cancer drugs," Annual Review of Medicine, vol. 63, pp. 185-198, 2012.

[13] K. Ahmad, "Gene delivery by nanoparticles offers cancer hope," The Lancet Oncology, vol. 3, no. 8, p. 451, 2002.

[14] R. Ramesh, "Nanoparticle-mediated gene delivery to the lung," Methods in Molecular Biology, vol. 434, pp. 301-331, 2008.

[15] D. Yuan, Y. Lv, Y. Yao et al., "Efficacy and safety of Abraxane in treatment of progressive and recurrent non-small cell lung cancer patients: a retrospective clinical study," Thoracic Cancer, vol. 3, no. 4, pp. 341-347, 2012.

[16] R. M. Schiffelers, J. M. Metselaar, M. H. A. M. Fens, A. P. C. A. Janssen, G. Molema, and G. Storm, "Liposome-encapsulated prednisolone phosphate inhibits growth of established tumors in mice," Neoplasia, vol. 7, no. 2, pp. 118-127, 2005.

[17] S. Simões, A. Filipe, H. Faneca et al., "Cationic liposomes for gene delivery," Expert Opinion on Drug Delivery, vol. 2, no. 2, pp. 237-254, 2005.

[18] M. Adamina, U. Guller, L. Bracci, M. Heberer, G. C. Spagnoli, and R. Schumacher, "Clinical applications of virosomes in cancer immunotherapy," Expert Opinion on Biological Therapy, vol. 6, no. 11, pp. 1113-1121, 2006.

[19] L. H. Lindner, M. E. Eichhorn, H. Eibl et al., "Novel temperature-sensitive liposomes with prolonged circulation time," Clinical Cancer Research, vol. 10, no. 6, pp. 2168-2178, 2004.

[20] L. Krishnan, L. Deschatelets, F. C. Stark, K. Gurnani, and G. D. Sprott, "Archaeosome adjuvant overcomes tolerance to tumorassociated melanoma antigens inducing protective CD8+ T cell responses," Clinical and Developmental Immunology, vol. 2010, Article ID 578432, 13 pages, 2010.

[21] X. Yao, K. Panichpisal, N. Kurtzman, and K. Nugent, "Cisplatin nephrotoxicity: a review," American Journal of the Medical Sciences, vol. 334, no. 2, pp. 115-124, 2007.

[22] T. Boulikas, "Low toxicity and anticancer activity of a novel liposomal cisplatin (Lipoplatin) in mouse xenografts," Oncology Reports, vol. 12, no. 1, pp. 3-12, 2004.

[23] P. Devarajan, R. Tarabishi, J. Mishra et al., "Low renal toxicity of lipoplatin compared to cisplatin in animals," Anticancer Research, vol. 24, no. 4, pp. 2193-2200, 2004.

[24] "Liposomal cisplatin (Nanoplatin) for advanced non-small cell lung cancer-first line (2012)," http://www.hsc.nihr.ac.uk/ topics/liposomal-cisplatin-nanoplatin-for-advanced-non-sm/.

[25] X. Wang, J. Zhou, Y. Wang et al., "A phase I clinical and pharmacokinetic study of paclitaxel liposome infused in nonsmall cell lung cancer patients with malignant pleural effusions," European Journal of Cancer, vol. 46, no. 8, pp. 1474-1480, 2010.

[26] J. Zhou, W. Y. Zhao, X. Ma et al., "The anticancer efficacy of paclitaxel liposomes modified with mitochondrial targeting conjugate in resistant lung cancer," Biomaterials, vol. 34 , no. 14 , pp. 3626-3638, 2013.

[27] S. Koudelka and J. Turánek, "Liposomal paclitaxel formulations," Journal of Control Release, vol. 163, no. 3, pp. 322-334, 2012.

[28] G. P. Stathopoulos, D. Antoniou, J. Dimitroulis et al., "Liposomal cisplatin combined with paclitaxel versus cisplatin and paclitaxel in non-small-cell lung cancer: a randomized phase III multicenter trial," Annals of Oncology, vol. 21, no. 11, pp. 22272232, 2010.

[29] S. North and C. Butts, "Vaccination with BLP25 liposome vaccine to treat non-small cell lung and prostate cancers," Expert Review of Vaccines, vol. 4, no. 3, pp. 249-257, 2005.
[30] G. T. Wurz, A. M. Gutierrez, B. E. Greenberg et al., "Antitumor effects of L-BLP25 Antigen-Specific tumor immunotherapy in a novel human MUC1 transgenic lung cancer mouse model," Journal of Translational Medicine, vol. 11, no. 1, pp. 64-77, 2013.

[31] Y.-L. Wu, K. Park, R. A. Soo et al., "INSPIRE: a phase III study of the BLP25 liposome vaccine (L-BLP25) in Asian patients with unresectable stage III non-small cell lung cancer," BMC Cancer, vol. 11, article 430, 2011.

[32] C. Lu, D. J. Stewart, J. J. Lee et al., "Phase I clinical trial of systemically administered TUSC2(FUS1)-nanoparticles mediating functional gene transfer in humans," Plos One, vol. 7, no. 4, Article ID e34833, 2012.

[33] S. H. Choi, S.-E. Jin, M.-K. Lee et al., "Novel cationic solid lipid nanoparticles enhanced p53 gene transfer to lung cancer cells," European Journal of Pharmaceutics and Biopharmaceutics, vol. 68, no. 3, pp. 545-554, 2008.

[34] K. H. Bae, J. Y. Lee, S. H. Lee, T. G. Park, and Y. S. Nam, "Optically traceable solid lipid nanoparticles loaded with siRNA and paclitaxel for synergistic chemotherapy with in situ imaging," Advanced Healthcare Materials, vol. 2, no. 4, pp. 576-584, 2013.

[35] A. Z. Wang, K. Yuet, L. Zhang et al., "ChemoRad nanoparticles: a novel multifunctional nanoparticle platform for targeted delivery of concurrent chemoradiation," Nanomedicine, vol. 5, no. 3, pp. 361-368, 2010.

[36] J. Jung, S. J. Park, H. K. Chung et al., "Polymeric nanoparticles containing taxanes enhance chemoradiotherapeutic efficacy in non-small cell lung cancer," International Journal of Radiation Oncololgy Biology Physics, vol. 84, pp. e77-e83, 2012.

[37] D.-W. Kim, S.-Y. Kim, H.-K. Kim et al., "Multicenter phase II trial of Genexol-PM, a novel Cremophor-free, polymeric micelle formulation of paclitaxel, with cisplatin in patients with advanced non-small-cell lung cancer," Annals of Oncology, vol. 18, no. 12, pp. 2009-2014, 2007.

[38] "A Phase II Trial of Genexol-PM and Gemcitabine in Patients With Advanced Non-small-cell Lung Cancer," 2013, http:// clinicaltrials.gov/show/NCT01770795.

[39] L. Jiang, X. Li, L. Liu, and Q. Zhang, “Thiolated chitosanmodified PLA-PCL-TPGS nanoparticles for oral chemotherapy of lung cancer," Nanoscale Research Letters, vol. 8, no. 1, p. 66, 2013.

[40] T. Zhao, H. Chen, L. Yang et al., "DDAB-modified TPGS-b(PCL-ran-PGA) Nanoparticles as oral anticancer drug carrier for lung cancer chemotherapy," Nano, vol. 8, no. 2, Article ID 1350014, 10 pages, 2013.

[41] A. Mehrotra, R. C. Nagarwal, and J. K. Pandit, "Lomustine loaded chitosan nanoparticles: characterization and in-vitro cytotoxicity on human lung cancer cell line L132," Chemical and Pharmaceutical Bulletin, vol. 59, no. 3, pp. 315-320, 2011.

[42] R. Liu, O. V. Khullar, A. P. Griset et al., "The pax-eNP placed at the time of surgical resection delayed local tumor recurrence and modestly prolonged survival in a murine Lewis lung carcinoma recurrence model," Annals of Thoracic Surger, vol. 91, no. 4, pp. 1077-1083, 2011.

[43] R. Yang, S.-G. Yang, W.-S. Shim et al., "Lung-specific delivery of paclitaxel by chitosan-modified PLGA nanoparticles via transient formation of microaggregates," Journal of Pharmaceutical Sciences, vol. 98, no. 3, pp. 970-984, 2009.

[44] K. Tahara, H. Yamamoto, N. Hirashima, and Y. Kawashima, "Chitosan-modified poly(d,l-lactide-co-glycolide) nanospheres for improving siRNA delivery and gene-silencing effects," European Journal of Pharmaceutics and Biopharmaceutics, vol. 74, no. 3, pp. 421-426, 2010. 
[45] C.-L. Tseng, T.-W. Wang, G.-C. Dong et al., "Development of gelatin nanoparticles with biotinylated EGF conjugation for lung cancer targeting," Biomaterials, vol. 28, no. 27, pp. 39964005, 2007.

[46] C.-L. Tseng, W.-Y. Su, K.-C. Yen, K.-C. Yang, and F.-H. Lin, "The use of biotinylated-EGF-modified gelatin nanoparticle carrier to enhance cisplatin accumulation in cancerous lungs via inhalation," Biomaterials, vol. 30, no. 20, pp. 3476-3485, 2009.

[47] S. Karthikeyan, N. R. Prasad, A. Ganamani, and E. Balamurugan, "Anticancer activity of resveratrol-loaded gelatin nanoparticles on NCI-H460 non-small cell lung cancer cells," Biomedicine and Preventve Nutrition, vol. 3, no. 1, pp. 64-73, 2013.

[48] J. Liu, J. Liu, L. Chu et al., "Novel peptide-dendrimer conjugates as drug carriers for targeting nonsmall cell lung cancer," International Journal of Nanomedicine, vol. 6, no. 1, pp. 59-69, 2010.

[49] G. M. Ryan, L. M. Kaminskas, B. D. Kelly, D. J. Owen, M. P. McIntosh, and C. J. H. Porter, "Pulmonary administration of PEGylated polylysine dendrimers: absorption from the lung versus retention within the lung is highly size-dependent," Molecular Pharmaceutics, vol. 10, no. 8, pp. 2986-2995, 2013.

[50] "Dendrimers improve anticancer efficacy in lung metastasis model," 2013, http://www.starpharma.com/news/150.

[51] R. Yang, S.-G. Yang, W.-S. Shim et al., "Lung-specific delivery of paclitaxel by chitosan-modified PLGA nanoparticles via transient formation of microaggregates," Journal of Pharmaceutical Sciences, vol. 98, no. 3, pp. 970-984, 2009.

[52] W. P. Su, F. Y. Cheng, D. B. Shieh, C. S. Yeh, and W. C. Su, "PLGA nanoparticles codeliver paclitaxel and Stat 3 siRNA to overcome cellular resistance in lung cancer cells," International Journal of Nanomedicine, vol. 7, pp. 4269-4283, 2012.

[53] N. Karra, T. Nassar, A. N. Ripin et al., "Antibody conjugated PLGA nanoparticles for targeted delivery of paclitaxel palmitate: efficacy and biofate in a lung cancer mouse model," Small, 2013.

[54] J. Conde, G. Doria, and P. Baptista, "Noble metal nanoparticles applications in cancer," Journal of Drug Delivery, vol. 2012, Article ID 751075, 12 pages, 2012.

[55] G. Peng, U. Tisch, O. Adams et al., "Diagnosing lung cancer in exhaled breath using gold nanoparticles," Nature Nanotechnology, vol. 4, no. 10, pp. 669-673, 2009.

[56] J.-A. A. Ho, H.-C. Chang, N.-Y. Shih et al., "Diagnostic detection of human lung cancer-associated antigen using a gold nanoparticle-based electrochemical immunosensor," Analytical Chemistry, vol. 82, no. 14, pp. 5944-5950, 2010.

[57] O. Barash, N. Peled, U. Tisch, P. A. Bunn Jr., F. R. Hirsch, and H. Haick, "Classification of lung cancer histology by gold nanoparticle sensors," Nanomedicine: Nanotechnology, Biology, and Medicine, vol. 8, no. 5, pp. 580-589, 2012.

[58] Y.-H. Chen, C.-Y. Tsai, P.-Y. Huang et al., "Methotrexate conjugated to gold nanoparticles inhibits tumor growth in a syngeneic lung tumor model," Molecular Pharmaceutics, vol. 4, no. 5, pp. 713-722, 2007.

[59] T. Yokoyama, J. Tam, S. Kuroda et al., "Egfr-targeted hybrid plasmonic magnetic nanoparticles synergistically induce autophagy and apoptosis in non-small cell lung cancer cells," PLoS ONE, vol. 6, no. 11, Article ID e25507, 2011.

[60] L. L. Ma, J. O. Tam, B. W. Willsey et al., "Selective targeting of antibody conjugated multifunctional nanoclusters (nanoroses) to epidermal growth factor receptors in cancer cells," Langmuir, vol. 27 , no. 12 , pp. 7681-7690, 2011.
[61] B. Lkhagvadulam, J. H. Kim, I. Yoon, and Y. K. Shim, "Sizedependent photodynamic activity of gold nanoparticles conjugate of water soluble purpurin-18-N-methyl-D-glucamine," BioMed Research International, vol. 2013, Article ID 720579, 10 pages, 2013.

[62] R. Govender, A. Phulukdaree, R. M. Gengan, K. Anand, and A. A. Chuturgoon, "Silver nanoparticles of Albizia adianthifolia: the induction of apoptosis in human lung carcinoma cell line," Journal of Nanobiotechnology, vol. 11, no. 5, pp. 1477-3155, 2013.

[63] W. G. Zhou and W. Wang, "Synthesis of silver nanoparticles and their antiproliferation," The Oriental Journal of Chemistry, vol. 28, no. 2, pp. 651-655, 2012.

[64] R. Foldbjerg, D. A. Dang, and H. Autrup, "Cytotoxicity and genotoxicity of silver nanoparticles in the human lung cancer cell line, A549," Archives of Toxicology, vol. 85, no. 7, pp. 743750, 2011.

[65] T. Sadhukha, T. S. Wiedmann, and J. Panyam, "Inhalable magnetic nanoparticles for targeted hyperthermia in lung cancer therapy," Biomaterials, vol. 34, no. 21, pp. 5163-5171, 2013.

[66] Y. Wang, Y. Zhang, Z. Du, M. Wu, and G. Zhang, “Detection of micrometastases in lung cancer with magnetic nanoparticles and quantum dots," International Journal of Nanomedicine, vol. 7, pp. 2315-2324, 2012.

[67] K. Li, B. Chen, L. Xu et al., "Reversal of multidrug resistance by cisplatin-loaded magnetic $\mathrm{Fe}_{3} \mathrm{O}_{4}$ nanoparticles in A549/DDP lung cancer cells in vitro and in vivo," International Journal of Nanomedicine, vol. 8, pp. 1867-1877, 2013.

[68] W. Sun, N. Fang, B. G. Trewyn et al., "Endocytosis of a single mesoporous silica nanoparticle into a human lung cancer cell observed by differential interference contrast microscopy," Analytical and Bioanalytical Chemistry, vol. 391, no. 6, pp. 21192125, 2008.

[69] A. J. Di Pasqua, M. L. Miller, X. Lu, L. Peng, and M. Jay, "Tumor accumulation of neutron-activatable holmiumcontaining mesoporous silica nanoparticles in an orthotopic non-small cell lung cancer," Inorgica Chimca Acta, vol. 393, no. 1, pp. 334-336, 2012.

[70] O. Taratula, O. B. Garbuzenko, A. M. Chen, and T. Minko, "Innovative strategy for treatment of lung cancer: targeted nanotechnology-based inhalation co-delivery of anticancer drugs and siRNA," Journal of Drug Targeting, vol. 19, no. 10, pp. 900-914, 2011.

[71] S. Sundarraj, "EGFR antibody conjugated mesoporous silica nanoparticles for cytosolic phospholipase A2 $\alpha$ targeted nonsmall lung cancer therapy," Journal of Cell Science and Therapy, vol. 3, no. 7, 2012.

[72] H. Maeda, "The enhanced permeability and retention (EPR) effect in tumor vasculature: the key role of tumor-selective macromolecular drug targeting," Advances in Enzyme Regulation, vol. 41, pp. 189-207, 2001.

[73] N. Dinauer, S. Balthasar, C. Weber, J. Kreuter, K. Langer, and H. Von Briesen, "Selective targeting of antibody-conjugated nanoparticles to leukemic cells and primary T-lymphocytes," Biomaterials, vol. 26, no. 29, pp. 5898-5906, 2005.

[74] H. Schreier, L. Gagné, T. Bock et al., "Physicochemical properties and in vitro toxicity of cationic liposome cDNA complexes," Pharmaceutica Acta Helvetiae, vol. 72, no. 4, pp. 215-223, 1997.

[75] G. Bao, S. Mitragotri, and S. Tong, "Multifunctional nanoparticles for drug delivery and molecular imaging," Annual Review of Biomedical Engineering, vol. 15, pp. 253-282, 2013.

[76] R. Wang, P. S. Billone, and W. M. Mullet, "Nanomedicine in action: an overview of cancer nanomedicine on the market and 
in clinical trials," Journal of Nanomaterials, vol. 2013, Article ID 629681, 12 pages, 2013.

[77] A. F. Hubbs, R. R. Mercer, S. A. Benkovic et al., "Nanotoxicology—a pathologist's perspective," Toxicologic Pathology, vol. 39, no. 2, pp. 301-324, 2011.

[78] J. McCarthy, I. Inkielewicz-Stepmiak, J. J. Corbalan, and M. W. Radomski, "Mechanisms of toxicity of amorphous silica nanoparticles on human using submucosal cells in vitro: protective effects of fisetin," Chemical Research in Toxicology, vol. 25, no. 10, pp. 2227-2235, 2012.

[79] B. Trouiller, R. Reliene, A. Westbrook, P. Solaimani, and R. H. Schiestl, "Titanium dioxide nanoparticles induce DNA damage and genetic instability in vivo in mice," Cancer Research, vol. 69, no. 22, pp. 8784-8789, 2009.

[80] A. Make, L. Wang, and Y. Rojanasakul, "Mechanisms of nanoparticle-induced oxidative stress and toxicity," BioMed Research International, vol. 2013, Article ID 942916, 15 pages, 2013.

[81] D. F. Emerich and C. G. Thanos, "The pinpoint promise of nanoparticle-based drug delivery and molecular diagnosis," Biomolecular Engineering, vol. 23, no. 4, pp. 171-184, 2006.

[82] S. M. Moghimi, A. C. Hunter, and J. C. Murray, "Longcirculating and target-specific nanoparticles: theory to practice," Pharmacological Reviews, vol. 53, no. 2, pp. 283-318, 2001.

[83] M. A. Dobrovolskaia, P. Aggarwal, J. B. Hall, and S. E. McNeil, "Preclinical studies to understand nanoparticle interaction with the immune system and its potential effects on nanoparticle biodistribution," Molecular Pharmaceutics, vol. 5, no. 4, pp. 487495, 2008. 

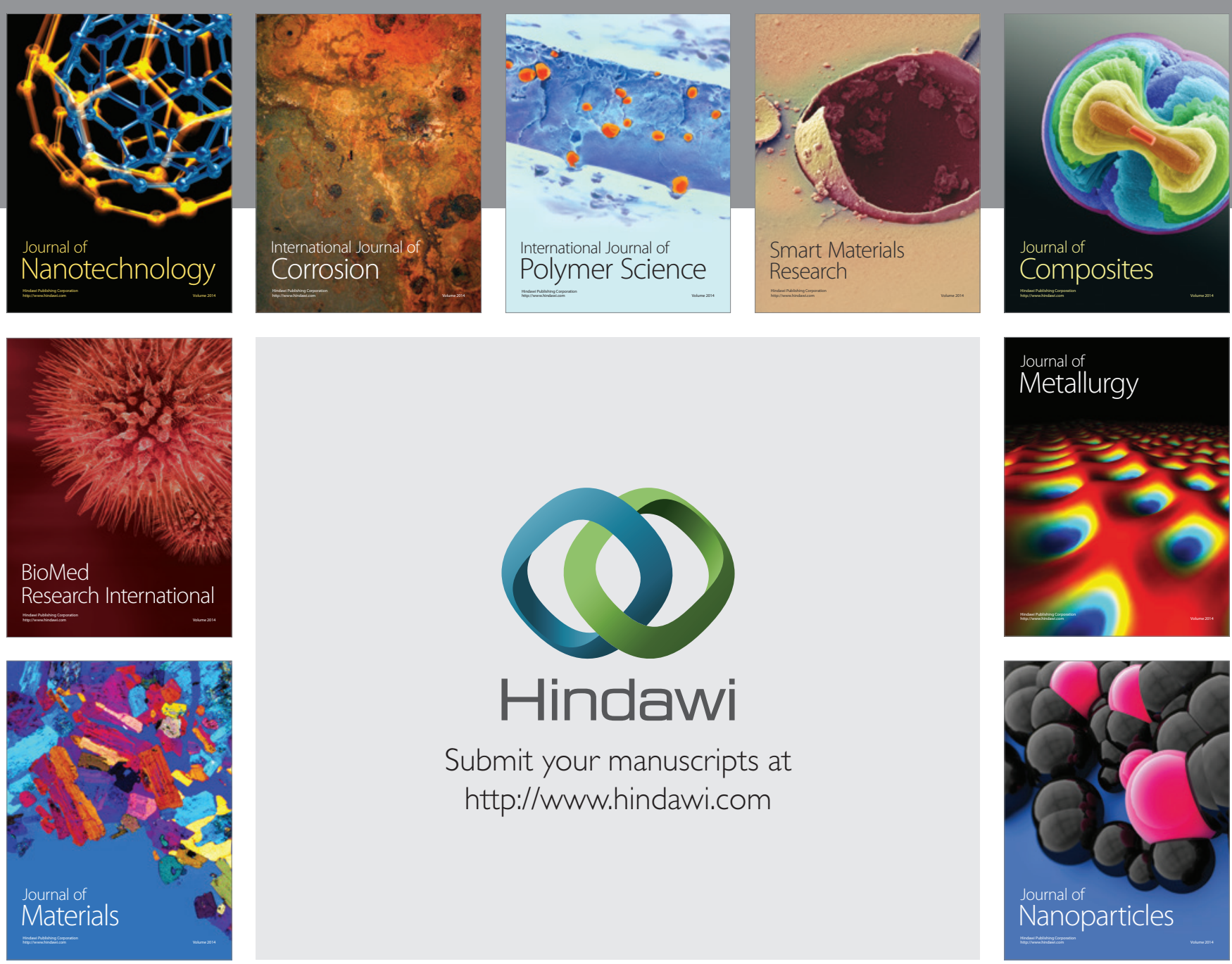

Submit your manuscripts at http://www.hindawi.com
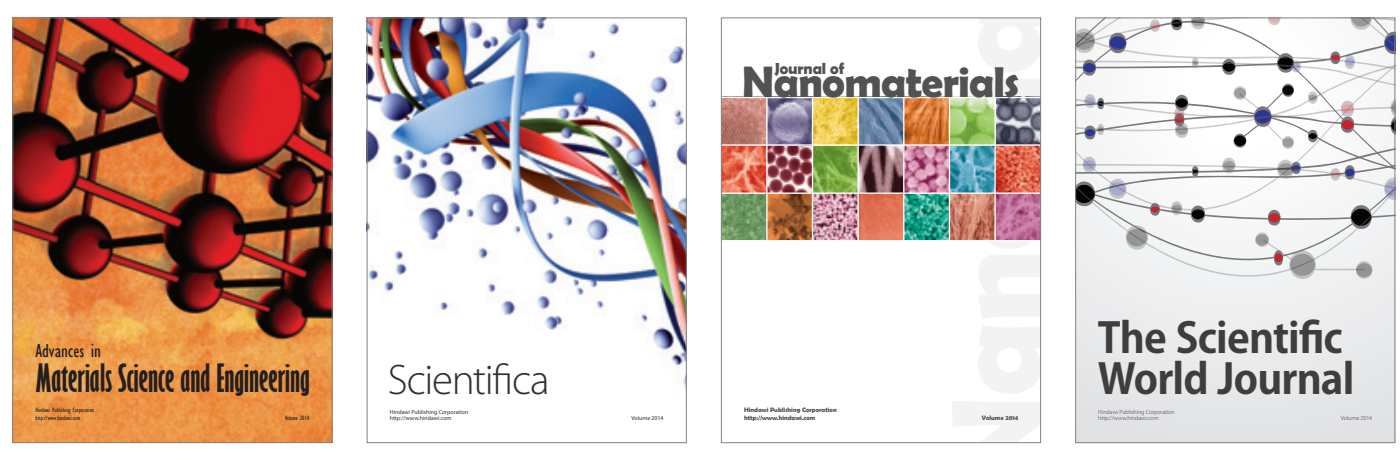

\section{The Scientific World Journal}
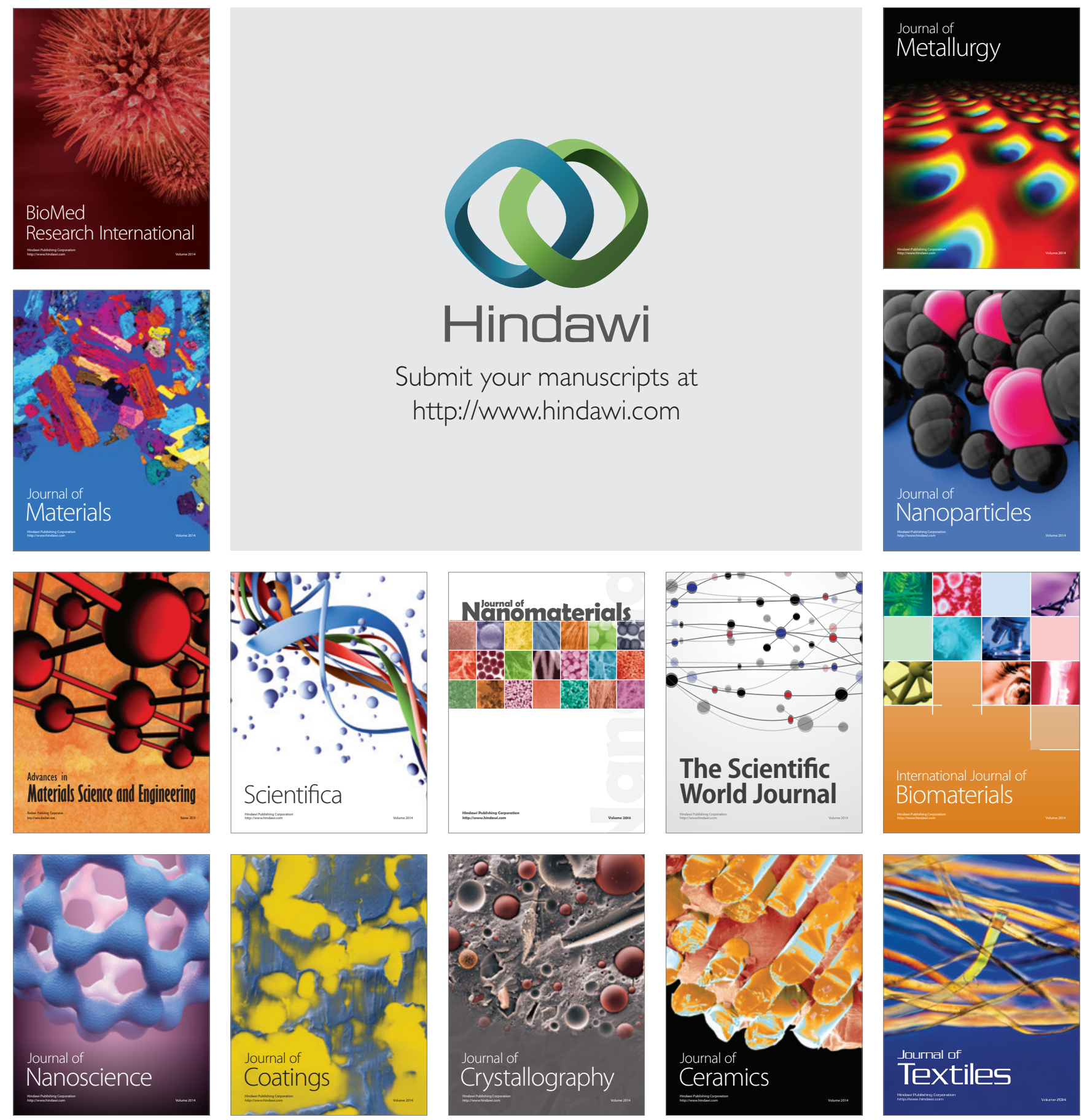